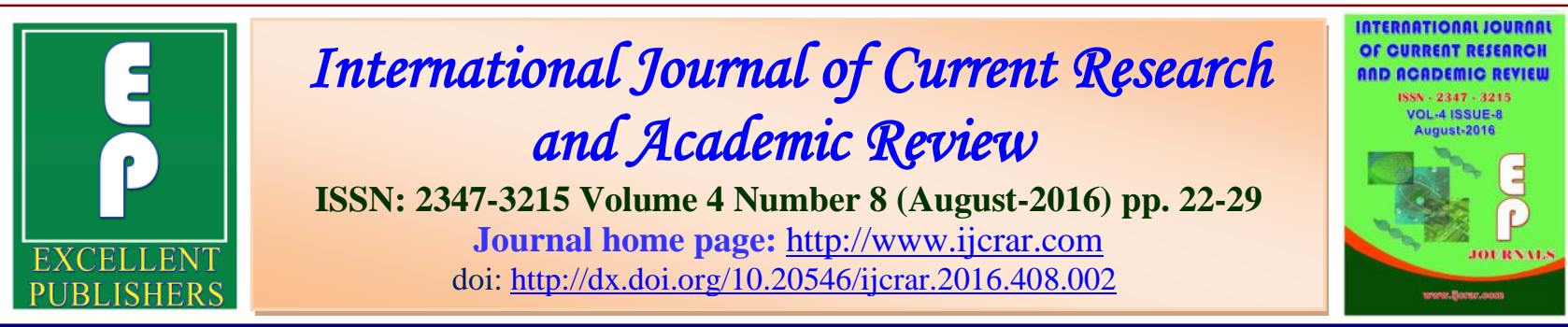

\title{
Prophylactic Screening Program of Children's Vision in the City of Pleven, Bulgaria
}

\author{
K.P. Valcheva*, S.V. Murgova and Ch.B. Balabanov \\ Clinic of Ophthalmology, University Hospital “Dr.GeorgiStranski”-Pleven, Bulgaria \\ *Corresponding author
}

\begin{tabular}{l|l}
\hline \multirow{2}{*}{$\begin{array}{l}\text { KEYWORDS } \\
\text { children, } \\
\text { visual acuity, } \\
\text { refractive error, } \\
\text { amblyopia. }\end{array}$} & $\begin{array}{l}\text { A B S T R A C T } \\
\text { Tofraction abnormalities in children } 3 \text { to } 6 \text { years of age in preschool within the } \\
\text { territory of Pleven. The children ages } 3 \text { to } 6 \text { that attend preschools in the } \\
\text { territory of Pleven who have passed through the free preventative eye } \\
\text { examination are 2332.The screening took place during October } 2013 \text { through }\end{array}$ \\
$\begin{array}{l}\text { January 2014. Every child had an observable decrease visual acuity were } \\
\text { asked to return for a comprehensive exam at the Ophthalmology Clinic at the }\end{array}$ \\
$\begin{array}{l}\text { University Hospital Dr. Georgi Stanski in Pleven. As a result of the } \\
\text { preventative exams of the } 2332 \text { responding and examining children, it can be } \\
\text { determined that there is a deviation from normal vision within 236 (10.1\%) } \\
\text { children. All of them were directed for a specialized visual exam.Fouty-four } \\
\text { children (18.6\%) showed up and induced a cycloplegia.Thirty-one } \\
\text { children(13.1\%)was written an optical correct. Within 18 (58.1\%) of these } \\
\text { children normal visual acuity was achieved with corrective lenses. However, } \\
\text { within } 12 \text { (38.7\%) children, due to amblyopia, the available corrective lenses } \\
\text { did not have the same result. Early detection and treatment of refractive errors } \\
\text { in children would lead to normal physical, neurological, and emotional } \\
\text { development. }\end{array}$
\end{tabular}

\section{Introduction}

A child's visual system is immature and malleable and undergoes intensive development during childhood. Vision in children gradually increases and reaches visual acuity of an adult around the age of 5 (Holladay, 2004; Pan et al., 2009). Many endogenous and exogenous factors can cause deviation in normal development of a child's vision. Reduced visual acuity (especially in a single eye) is the reason for the development of lasting amblyopia, which children are not aware of because the other eye compensates for the imperfection. The result is decreased vision for life which can prevent the integration of the individual in society, as well as professional development. 


\section{Materials and Methods}

During the period of October 2013 through January 2014, children ages 3 to 6 were examined within 23 childcare facilities and preschools in the city and territory of Pleven.

Parents were given questionnaires asking to provide information on past and present eye disease, family history, and harmful habits.

\section{Preventive Examination}

Children were passed preventive examinations on site in the kindergartens for detecting an ocularpathology, including: view, study of ortoptic status, illumination of transparent eye medium (fundus reflex).

Visual acuity was tested using a children cardboard visual table with pictures of UNIMED PHARMA placed at a 3 meter distance. The normal accepted visusis $\geq 0.8$ pereye.

\section{Comprehensive Examination}

Those children who did not respond to the visual acuity capturing as well as those with below normal visus were asked to come to the eye clinic at the University Hospital "Dr. GeorgiStranski"-Pleven.

The children that came to the clinic were given a visual acuity exam of the light table with mirror at 2.5 meters from it. Those with reduced visual acuity we induced cycloplegia (Cyclogyl 3X every 15 minutes) and subsequent auto refractometry was measured with ARM ChaRops CRK 7000. Following the exam glasses were prescribed and the vision was tested again.

The data from the exams was statistically mapped out and analyzed using Microsoft Excel.

\section{Results and Discussion}

Of the 2332 children surveyed and examined 1174 were female $(50.3 \%)$ and 1158 were male $(49.7 \%)$.

Thenumberofchildrenwhohavevisitedanopht halmologistbeforeourpreventativeprogramw ere 532(22.8\%). Of those, $218(41 \%)$ were directed by general physician, 30(5.6\%), by a pediatrician, and 284 children $(53.4 \%)$ visited an eye doctor at the discretion of their parents. Children with optical correction were 26 (4.9\%). The remaining 1800 children $(77.2 \%)$ were not examined by the ophthalmologist at this point (Figure $1)$.

A prophylactic review revealed that 2096 children (89.9\%) had normal visual acuity $\geq 0.8$ in each eye.Deviation in the vision was observed in 236 children (10.1\%). Visual acuity under 0.8 in one eye was observed in 67 children $(28.4 \%)$, and decreased vision in both eyes in 96 children (40.7\%). In addition, 73 children (30.9\%) did not cooperate in the capture of the visual acuity.

Children with low vision under 0.8 and those who did not get examined were summoned to the eye clinic.Fourty-four children came to the clinic and were given a comprehensive exam $(18.6 \%$ of the summoned 236 children for controlled exam at the clinic). Data from the auto refractometer for their objective refraction after cycloplegia shows that the most common refraction anomalyamong the children is astigmatism (32 children/72.7\%), followed by hypermetropia (11 children $125 \%$ ), and least common being myopia (1 child/2.3\%) (Figure 2).

Hypermetropic astigmatism was found in 22 children $(68.8 \%)$, myopic astigmatism - in 6 
children (18.7\%), and mixed astigmatism in 4 children $(12.5 \%)$ (Figure 3$)$.

Low level hypermetropia (under $+2.0 \mathrm{dsph}$ ) was observed in $7(63.6 \%)$ children, midlevel (between +2.0 dsphand $+5 \mathrm{dsph}$ ) in $4(36.4 \%)$ children, while high level (above $+5.0 \mathrm{dsph}$ ) was not observed among the children. Low level myopia (under -3.0 dsph) was observed in one child, mid level (between -3.0 dsph and -6.0 dsph) and high (above $-6.0 \mathrm{dsph}$ ) level was not found in the examined children (Figure 4).

At the clinic, 31 children $(70.5 \%$ of the children with refractive errors identified on the basis of the objective auto refractometry) were prescribed glasses after the constriction of the pupils ( 5 to 7 days after cycloplegia). The remaining 13 children $(29.5 \%)$ with identified refraction anomaly did not come forward for their prescription glasses.

Already during the prescription of optical correction, 18 children $(58.1 \%)$ improved their eyesight with glasses to $0.8-1.0,12$ children $(38.7 \%)$ did not achieve visual acuity $0.8-1.0$ despite the correction, and 1 child $(3.2 \%)$ did not respond in the examination of visual acuity and the correction was prescribed at the discretion of the physician.

Amblyopia was observed among 12 children $(38.7 \%)$. Of these children amblyopia in one eye - among 4 children(33.3\%), and in two eyes -8 children $(66.7 \%)$ (Figure 5 ).

Types of refraction errors among children with double amblyopia are as follows: hypermetropia -among $5(62.5 \%)$ children, myopicastigmatism - among 2 (25\%) children, and mixed astigmatism - within 1 $(12.5 \%)$ child. For amblyopia in a single eye, the distribution is the following: 2
$(50 \%)$ children with hypermetropia, 1(25\%) child with myopic astigmatism, and $1(25 \%)$ child with mixed astigmatism (Figure 6).

In children with amblyopia in one eye, 1 $(25 \%)$ of them had visual deviation of the eye (ezotropia). Anisometropia was also found in one child (25\%) of those established to have amblyopia in one eye. There were no other congenital or acquired diseases (disorders) of the visual system amnog the examined children.

Preschool vision screening programs has been introduced with the aim of detecting ophthalmic disorders at a younger age. The prompt and early treatment of ocular disorders is important to avoid lifelong visual impairment. Many countries have introduced guidelines for screening preschool children for refractive errors (Kontrova and Petrova, 2005; Ehrlich et al., 1983; Hard et al., 2002; Kassmann-Kellner and Ruprecht, 2000; Packwood et al., 1999). American Acadely of Pediatrics (AAP) and American Academy of Ophthalmologist (AAO) recommend assessment in the newborn period and then at all subsequent routine health visits (American Acadely of Pediatrics et al., 2003) Canadian Task Force on Preventive Health Care recommends ageappropriate evaluations of visual system(Canadian Task Force on Preventive Health Care, 2003).

In Bulgaria, there is no functioning system for the preventative examination of vision in children ages 3 to 6 and only $22.8 \%$ of the examined children have visited an ophthalmologist in the past. General physicians, pediatritions, and parents are those who have a leading role in preserving a child's vision. 


\section{Int.J.Curr.Res.Aca.Rev.2016; 4(8): 22-29}

Fig.1 Distribution of children who have been examined by ophthalmologist by this point and those who have been selected for examination

- Not examined by an eye doctor $\mathbf{D i r e c t e d}$ by general physician

Directed by pediatrician $\quad$ Discretion of the parents

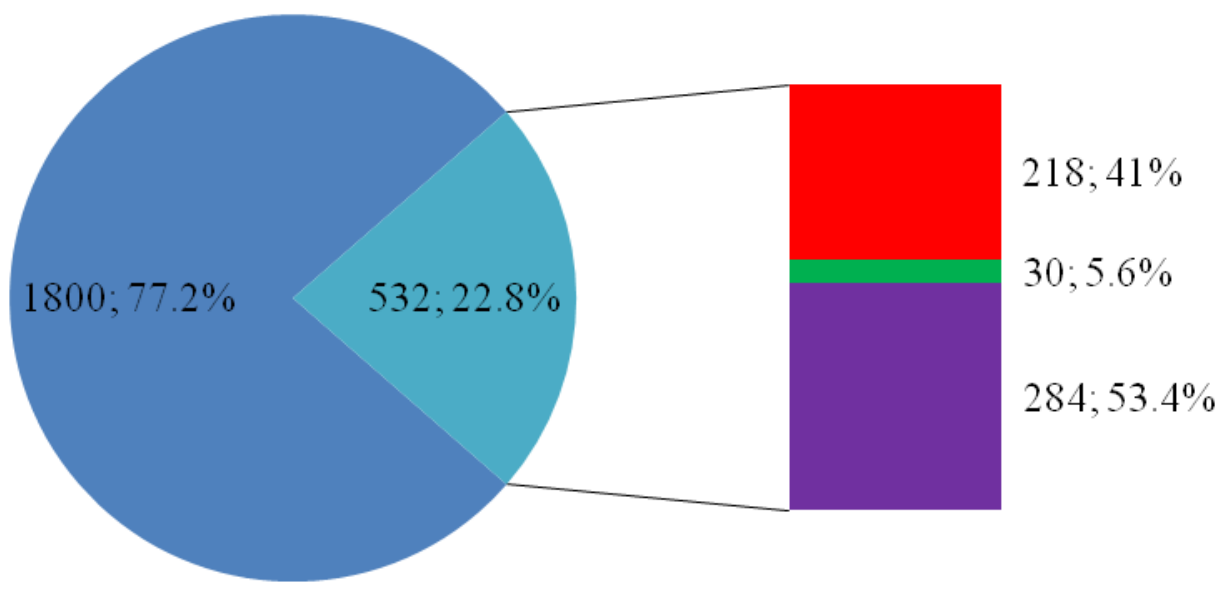

Fig.2 Distribution of types of refraction errors among children

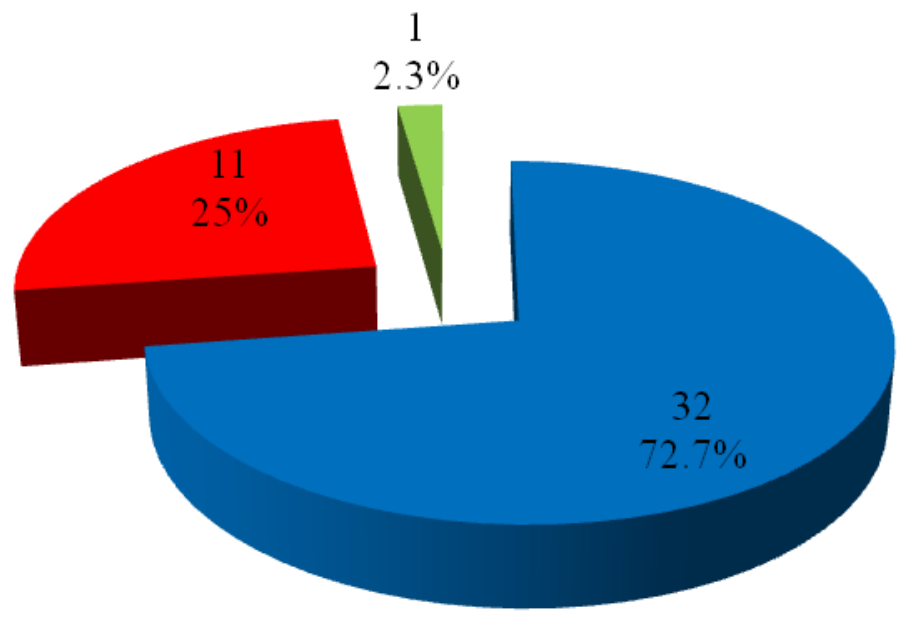

- Astigmatism

- Hypermetropia

Myopia 
Int.J.Curr.Res.Aca.Rev.2016; 4(8): 22-29

Fig.3 Distribution by type of astigmatism among children

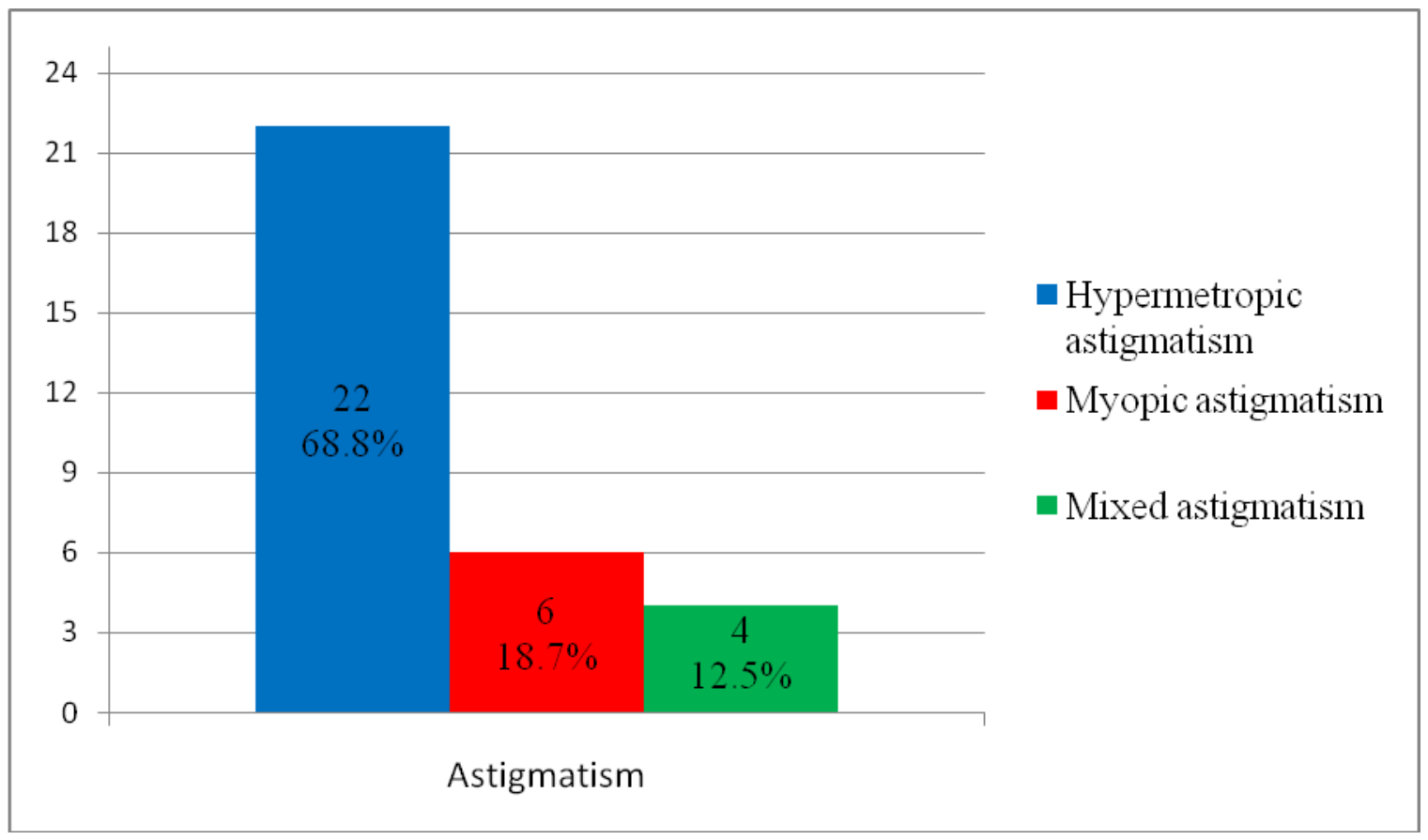

Fig.4 Distribution of levels of hypermetropia and myopia among children

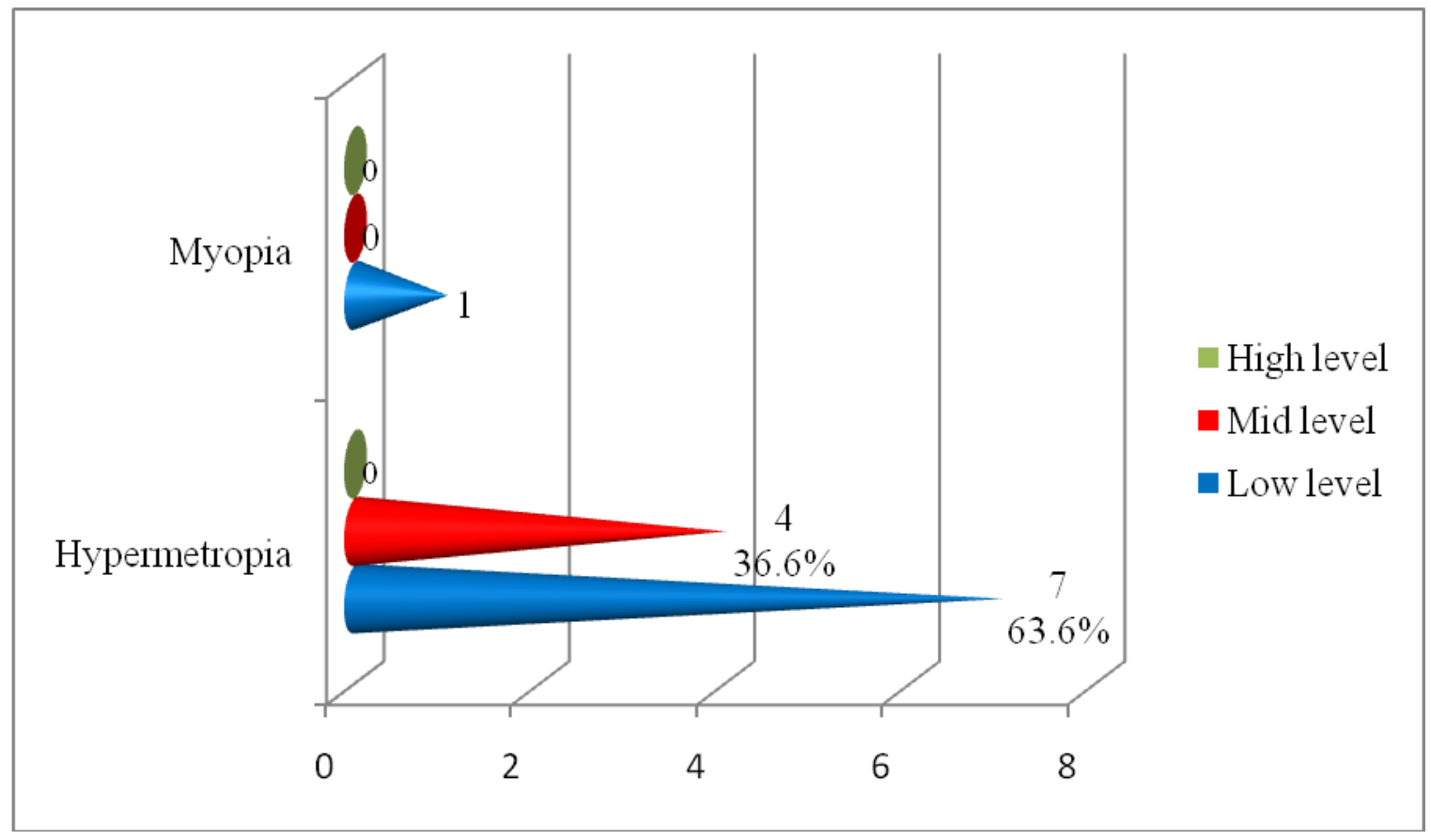


Fig.5 Distribution of amblyopia within one or two eyes among children

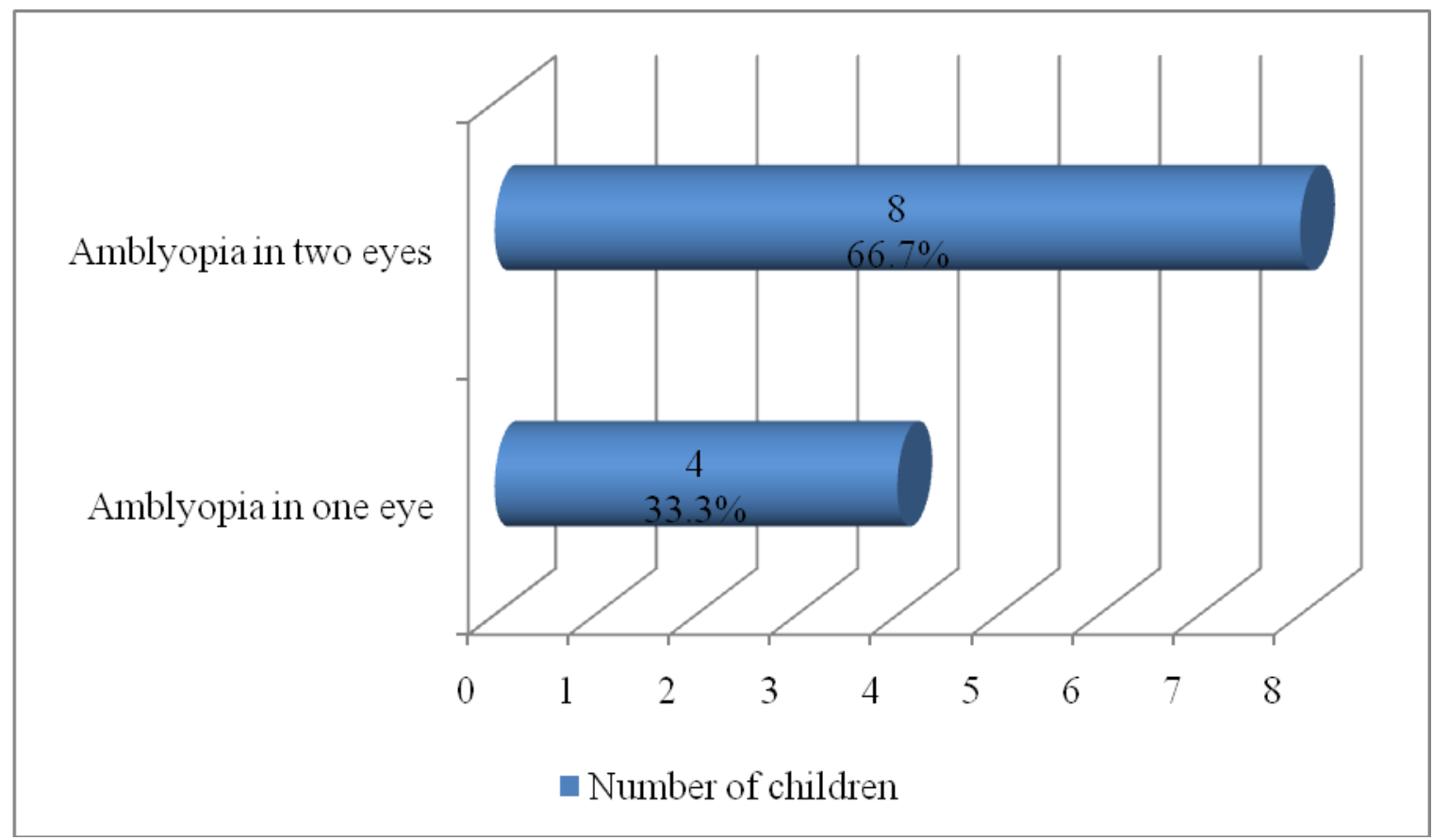

Fig.6 Distribution of amblyopia by the type of the refraction anomaly among children

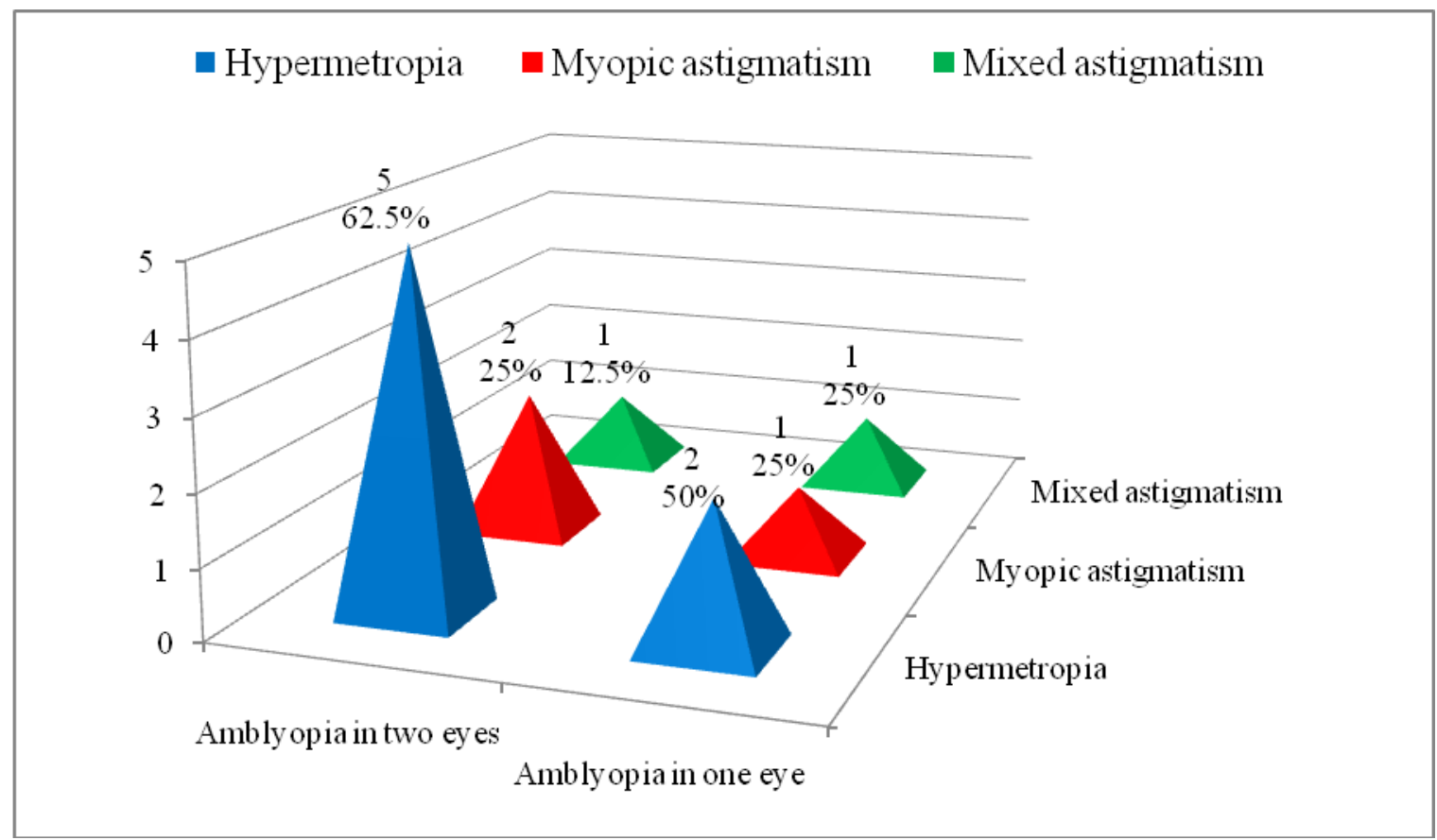

The prophylactic review revealed236 children with decreased vision, but only 44 of them were re-examined at the Eye
clinic.This can be explained by an insufficient health culture of the parents or seekinghelp at other eye specialists. 
Int.J.Curr.Res.Aca.Rev.2016; 4(8): 22-29

Refraction anomalies are the most common cause for visual problems in children (Agency for Healthcare Research and Quality, 2011). In our study, $1.89 \%$ of children have refraction errors. Astigmatism is most commonly $(72.7 \%)$ andthis corresponds to data found in the literature on the topic.

Refraction errors are the main reason for the development of amblyopia. Amblyopiais the leading cause of decreased vision of the eye among people ages 20-70 (National Eye Institute Office of Biometry and Epidemiology,1984). Amblyopia is the first leading cause of blindness of the one eye among children certificated in Regional expert medical advisory committees and the second leader among children examined by Children ocular cabinet and Eye Clinic in the city of Pleven (Stateva et al., 2005).

The most common cause of amblyopiainthe one eye is strabismus and/oranisometropia, while amblyopiain the two eyes is most commonly the result of refraction anomaly in both eyes (Tarczy-Hornoch et al., 2011). Amblyopiadue to strabismus is determined by the parents and general physicians but the cause of the difference in refraction between the two eyes is not demonstrated and is often left unrecognized(Von Noorden, 1990; Flynn et al.,1999).

\section{Conclusion}

Refraction errors are the main reason for permanently reduced vision and disability of children in the future. This condition is reversible if it is treat in a timely manner. Refraction anomalies are necessary to be diagnosed in time. Therefore, is appropriate to have a functional system for prophylactic examinations for children ages 3-6. This is the most effective way to improve the vision of children for their whole life.

\section{References}

Agency for Healthcare Research and Quality. Evidence synthesis number 81.Screening for visual impairment in children ages 1-5 years:systematic review to update the 2004 U.S.Preventive Services Task Force Recommendation. Aviable at: www.uspreventiveservicestaskforce.o rg/uspstfl1/vischildren/vischildes.pdf Accessed October 31,2011.

American Academy of Pediatrics. 2003. Committee on Practice and Ambulatory Medicine and Section on Ophthalmology; American Association of Certified Orthoptists; American Association for Pediatric Ophthalmology and Strabismus; American Acad. Ophthalmol., Policy Statement: Eye Examination in Infants, Children, and Young Adults by Pediatricians. Pediatrics. 111(4): 902-907.

Canadian Task Force on Preventive Health Care. New grades for recommendations from the Canadian Task Force on Preventive Health Care. CMAJ, 169: 207-8.

Ehrlich, M.I., Reinecke, R.D., Simons, K. 1983. Preschool vision screening for amblyopia and strabismus: programs, methods, guidelines. Surv Ophthalmol., 28: 145-63.

Flynn, J.T., Woodruff, G., Thompson, J.R., Hiscox, F., Feuer, W., Schiffman, F., Corona, A., Smith, L.K.1999. The therapy of amblyopia: an analysis comparing the results of amblyopia therapy utilizing two pooled data sets. Trans Am Ophthalmol Soc., 97: 373390. 
Hard, A., Sjodell, L., Borres, M.P et al.2002. Preschool vision screening in a Swedish city region: results after alteration of criteria for referral to eye clinics. Acta Ophthalmologica Scandinavica, 80: 608-11.

Holladay, J.T. 2004. Visual acuity measurements. J Cataract Refract Surg, 30(2): 287-290.

Kassmann-Kellner, B., Ruprecht, K.W.2000. Vision screening survey of all children starting primary school in 1998 in the federal state of Saarland, Germany. Strabismus, 8: 201-7.

Kontrova, E., Petrova, G. 2005. Prevention of child hood vision in the context of the global initiative "Vision2020". Reference newsletter of Ophthalmol., 3: 19-24.

National Eye Institute Office of Biometry and Epidemiology: Report on the National Eye Institute's Visual Acuity Impairment Survey Pilot Study. Washington, DC: US Department of Health and Human Services, 1984.

Packwood, P.A., Cruz, O.A., Rychwalski, P.J et al. 1999. The psychosocial effects of amblyopia study. J. AAPOS, 3: 15-17.

Pan, Y.,Tarczy-Hornoch, K., Cotter, S.A et al.2009. Visual acuity norms in preschool children: the Multi-Ethnic Pediatric Eye Disease Study. Optom. Vis. Sci., 86(6): 607-12.

Stateva, D., Balabanov, Ch., Velkova, A., Krivoshiyska, E., Statev, K. 2005. Retrospective study of prevalence of blindness among children, according to the Eye Clinic, Children's eye Cabinetand REMC- Pleven. Trakia J Sci., 3(1): 93-95.

Tarczy-Hornoch, K., Varma, R., Cotter, S.A., McKean-Cowdin, R., Lin, J.H., Borchert, M.S et al.2011. Risk factors for decreased visual acuity in preschool children: the multi-ethnic pediatric eye disease and Baltimore pediatric eye disease studies. Ophthalmol., 118(11): 2262-2273.

Von Noorden, G.K. 1990. Binocular Vision and Ocular Motility. $4^{\text {th }}$ edition. St Louis: Mosby.

\section{How to cite this article:}

Valcheva, K.P., S.V. Murgova and Balabanov, Ch.B. 2016. Prophylactic Screening Program of Children's Vision in the City of Pleven, Bulgaria. Int.J.Curr.Res.Aca.Rev.4(8): 22-29. doi: http://dx.doi.org/10.20546/ijcrar.2016.408.002 